hep-th/0605213

CAS-BHU/Preprint

\title{
A generalization of the horizontality condition in the superfield approach to nilpotent symmetries for QED with complex scalar fields
}

\author{
R.P.Malik ${ }^{*}$ \\ Centre of Advanced Studies, Physics Department, \\ Banaras Hindu University, Varanasi-221 005, India \\ E-mail address: malik@bhu.ac.in
}

\begin{abstract}
We provide a generalization of the horizontality condition of the usual superfield approach to Becchi-Rouet-Stora-Tyutin (BRST) formalism to obtain the (anti-)BRST symmetry transformations for all the fields of a four $(3+1)$-dimensional interacting 1-form $U(1)$ gauge theory (QED) within the framework of the augmented superfield formalism. In the above interacting gauge theory, there is an explicit coupling between the 1-form $U(1)$ gauge field and the complex scalar fields. This interacting gauge field theory is considered on the (4,2)-dimensional supermanifold parametrized by the four even spacetime variables $x^{\mu}$ (with $\mu=0,1,2,3$ ) and a pair of odd Grassmannian variables $\theta$ and $\bar{\theta}$. The above nilpotent (anti-)BRST symmetry transformations are obtained due to the imposition of a gauge (i.e. BRST) invariant restriction on the appropriate superfields defined on the $(4$, 2)-dimensional supermanifold. This restriction owes its origin to a pair of (super) covariant derivatives and their intimate connection with the 2-form (super) curvatures. The results obtained, due to the application of the horizontality condition alone, are contained in the results deduced due to the imposition of the above gauge invariant restriction.
\end{abstract}

PACS numbers: 11.15.-q; 12.20.-m; 03.70.+k

Keywords: Augmented superfield formalism; gauge (i.e. BRST) invariant restriction; QED with complex scalar fields in four dimensions; geometrical interpretations

${ }^{*}$ On leave of absence from S. N. Bose National Centre for Basic Sciences, Block-JD, Sector-III, Salt Lake, Kolkata-700 098, West Bengal, India. E-mail address: malik@bose.res.in 


\section{Introduction}

The usual superfield approach to Becchi-Rouet-Stora-Tyutin (BRST) formalism [1-6] provides a deep connection between some of the key mathematical properties associated with the (anti-)BRST symmetries (as well as corresponding generators) and the partial derivatives w.r.t. the Grassmannian variables of the superspace coordinates that characterize the $(D, 2)$-dimensional supermanifold on which a given $D$-dimensional $p$-form $(p=1,2,3 \ldots \ldots)$ (non-)Abelian gauge theory is considered. The above $(D, 2)$-dimensional supermanifold is parametrized by the number $D$ of the commuting spacetime variables $x^{\mu}$ (with $\mu=0,1,2 \ldots \ldots D-1$ ) and a pair of anticommuting (i.e. $\theta^{2}=\bar{\theta}^{2}=0, \theta \bar{\theta}+\bar{\theta} \theta=0$ ) Grassmannian variables $\theta$ and $\bar{\theta}$. On this supermanifold, a $(p+1)$-form super curvature is constructed with the help of the super exterior derivative $\tilde{d}=d x^{\mu} \partial_{\mu}+d \theta \partial_{\theta}+d \bar{\theta} \partial_{\bar{\theta}}$ (with $\tilde{d}^{2}=0$ ) and the super $p$-form gauge connection $\tilde{A}^{(p)}$. This is subsequently equated, due to the well-known horizontality condition [1-6], to the ordinary $(p+1)$-form curvature defined on the $D$-dimensional ordinary spacetime manifold with the help of the ordinary exterior derivative $d=d x^{\mu} \partial_{\mu}$ (with $d^{2}=0$ ) and the ordinary $p$-form gauge connection $A^{(p)}=\frac{1}{p !}\left(d x^{\mu_{1}} \wedge d x^{\mu_{2}} \ldots \ldots \wedge d x^{\mu_{p}}\right) A_{\mu_{1} \mu_{2} \ldots \ldots \mu_{p}}$ that defines the $p$-rank antisymmetric tensor gauge potential of the theory. The celebrated horizontality condition, christened as the soul-flatness condition in [7], mathematically amounts to setting equal to zero all the Grassmannian components of the $(p+1)$-rank (anti)symmetric tensor that constitutes the $(p+1)$-form super curvature defined on the above supermanifold.

The above horizontality condition has been extensively exploited to derive the nilpotent (anti-)BRST symmetry transformations for the gauge field and corresponding fermionic (anti-)ghost fields of the four $(3+1)$-dimensional (4D) 1-form (i.e. $A^{(1)}=d x^{\mu} A_{\mu}$ ) nonAbelian gauge theory. To be more specific, this $4 \mathrm{D}$ non-Abelian theory is first considered on a $(4,2)$-dimensional supermanifold parametrized by the four spacetime (even) coordinates $x^{\mu}(\mu=0,1,2,3)$ and a pair of Grassmannian (odd) variables $\theta$ and $\bar{\theta}$. An appropriate 2-form super curvature $\tilde{F}^{(2)}=\tilde{d} \tilde{A}^{(1)}+\tilde{A}^{(1)} \wedge \tilde{A}^{(1)}$ is constructed with the help of the super exterior derivative $\tilde{d}$ and super 1 -form connection $\tilde{A}^{(1)}$. This is equated to the ordinary 2form curvature $F^{(2)}=d A^{(1)}+A^{(1)} \wedge A^{(1)}$ constructed with the help of the ordinary exterior derivative $d=d x^{\mu} \partial_{\mu}$ and ordinary 4D 1-form connection $A^{(1)}$. This equality (i) leads to the derivation of nilpotent (anti-)BRST symmetry transformations, and (ii) provides the geometrical interpretation for the nilpotent (anti-)BRST charges (and the nilpotent symmetry transformations they generate) in the language of the translational generators along the Grassmannian directions of the (4, 2)-dimensional supermanifold. However, the latter type of geometrical interpretations remain confined only to the gauge field and the corresponding (anti-)ghost fields of the theory. The matter fields of the interacting 1form non-Abelian gauge theory remain untouched in the framework of the usual superfield formulation (with the theoretical arsenal of horizontality condition alone).

The above trick has also been exploited in the context of the derivation of the nilpotent 
(anti-)BRST symmetry transformations for the 2-form (i.e. $\left.A^{(2)}=\frac{1}{2 !}\left(d x^{\mu} \wedge d x^{\nu}\right) B_{\mu \nu}\right)$ noninteracting Abelian gauge theory in $4 \mathrm{D}$ where a 3 -form super curvature $\tilde{F}^{(3)}=\tilde{d} \tilde{A}^{(2)}$, constructed with the help of the super exterior derivative $\tilde{d}$ and super 2-form connection $\tilde{A}^{(2)}$, is equated to the ordinary $4 \mathrm{D} 3$-form $F^{(3)}=d A^{(2)}$ constructed with the help of the ordinary exterior derivative $d$ and 2-form ordinary connection $A^{(2)}$. As expected, here too, one obtains the nilpotent (anti-)BRST transformations for the second rank antisymmetric gauge field $B_{\mu \nu}$ and the corresponding (anti)commuting (anti-)ghost fields of the theory. Of course, the (anti-)ghost fields turn out to be bosonic as well as fermionic in nature for the 2-form Abelian gauge theory. Even for this Abelian theory, the matter fields are not discussed at all in the framework of the usual superfield approach to BRST formalism.

In a recent set of papers [8-17], the above horizontality condition of the usual superfield approach has been consistently extended so as to derive the nilpotent (anti-)BRST symmetry transformations for the matter fields together with the above nilpotent transformations associated with the gauge and (anti-)ghost fields. This extended version of the superfield formalism has been christened as the augmented superfield formalism where, in addition to the horizontality condition, some other physically interesting restrictions are imposed on the superfields of the appropriately chosen supermanifold. In the latter category of restrictions, mention can be made of the equality of (i) the conserved currents corresponding to the gauge symmetries [8-10,13], (ii) any conserved quantities for the reparametrization invariant theories $[11,12]$, and (iii) the gauge invariant quantities owing their origin to the covariant derivatives on the appropriately chosen superfields [14-17]. One obtains logically consistent nilpotent (anti-)BRST symmetry transformations for all the fields due to the application of the restrictions (i) and (ii). However, the application of the restriction (iii) on the superfields (defined on the appropriately chosen supermanifolds) leads to the derivation of mathematically exact nilpotent (anti-)BRST symmetry transformations for all the fields. In a very recent set of papers [18,19], the nilpotent (anti-)BRST symmetry transformations for all the fields of the interacting 4D (non-)Abelian gauge theories (with the Dirac fields as the interacting matter fields) have been derived from a single restriction on the appropriate superfields of the supermanifold. These attempts have been made to generalize the horizontality condition to obtain all the nilpotent (anti-)BRST symmetry transformations for all the fields of a given gauge theory without spoiling the geometrical interpretations of some of the key properties associated with the nilpotent (anti-)BRST symmetries (and corresponding nilpotent charges) that are provided by the horizontality condition alone. All the above mathematically consistent extensions of the usual superfield formalism are called by us as the augmented superfield approach to BRST formalism.

The central theme of our present paper is to demonstrate that the ideas of the augmented superfield formalism, with a single gauge (i.e. BRST) invariant restriction on the appropriately chosen superfields (defined on a suitable supermanifold) [18,19], can be extended to derive the on-shell as well as off-shell nilpotent (anti-)BRST symmetry transformations for all the fields of an interacting four $(3+1)$-dimensional $U(1)$ gauge theory where there 
is an explicit coupling between the $U(1)$ gauge field and the charged complex scalar fields. We show that all the results, obtained due to the application of the horizontality condition of the usual superfield formulation, are contained in the results deduced by exploiting our present gauge (i.e. BRST) invariant restriction on the appropriately chosen superfields. On top of it, the appropriate modifications of our present restriction on the superfields (defined on a suitably chosen supermanifold) provides a precise way to derive the on-shell nilpotent (anti-)BRST transformations for all the fields (including the matter fields) of the theory in a separate and independent manner. It should be re-emphasized that the horizontality condition alone does not shed any light on the derivation of the nilpotent symmetry transformations associated with the matter fields of any arbitrary interacting gauge theory in any arbitrary dimension of spacetime. Thus, our present endeavour is an important step in the direction to generalize the horizontality condition of the usual superfield approach to a more general condition on the appropriately chosen superfields. Our present Abelian gauge (i.e. BRST) invariant restriction owes its origin to a pair of (super) covariant derivatives, their operation on matter (super) fields and their intimate connection with the Abelian (super) curvature 2-forms defined on the appropriately chosen (super) spacetime manifolds. In our present investigation, as a warm up exercise, we first derive the on-shell nilpotent BRST and anti-BRST symmetry transformations for all the fields of the present interacting U(1) gauge theory by invoking the chiral and anti-chiral superfields defined on the (4, 1)-dimensional super sub-manifolds (cf. section 3 below). Later on, we merge together these superfields to obtain the general $(4,2)$-dimensional superfields for the derivation of the off-shell nilpotent (anti-)BRST symmetry transformations together for all the fields of our present gauge theory from a single restriction on the matter superfields (cf. section 4 below). Thus, our main results are contained in sections 3 and 4 .

The compelling reasons behind our present investigation are primarily four in number. First and foremost, it is important to generalize our ideas of the augmented superfield approach to BRST formalism $[18,19]$ to a new field theoretical system where a single gauge (i.e. BRST) invariant restriction on the superfields (defined on the appropriately chosen supermanifolds) leads to the derivation of the nilpotent (anti-)BRST symmetry for all the fields of the interacting gauge theory. In fact, the above ideas have been found to be true for the derivation of the nilpotent symmetry transformations in the cases of (i) an interacting 4D Abelian U(1) gauge theory where there is an explicit coupling between the gauge field and the Dirac (matter) fields, and (ii) an interacting 4D non-Abelian gauge theory where the Noether conserved current, constructed with the help of Dirac fields, couples to the $S U(N)$ non-Abelian gauge field. In our present endeavour, we exploit primarily the same restriction for a new field theoretic model where there is a coupling between the $U(1)$ gauge field and the Noether conserved current constructed with the help of the charged complex scalar fields and the gauge field itself. Thus, our present investigation is essential to put our earlier ideas on a firmer ground. Second, in our earlier works $[13,14]$, connected with the superfield approach to the derivation of nilpotent symmetry transformations for 
the complex scalar fields, we exploited two separate restrictions on the $(4,2)$-dimensional supermanifold which included the horizontality condition of the usual superfield formalism as one of the restrictions. Our present endeavour is more economical and aesthetically more appealing because we derive all the nilpotent (anti-)BRST symmetry transformations from a single gauge invariant restriction. Third, the single restriction exploited in our present investigation is physically more appealing because this restriction is a gauge (i.e. BRST) invariant condition on the suitably chosen superfields. In contrast, the horizontality condition is intrinsically a gauge covariant restriction on the gauge superfield. Finally, our present field theoretical model is (i) useful in the context of gauge theory of standard model, and (ii) different from the interacting 4D (non-)Abelian gauge theories with fermionic Dirac fields. For instance, the present model allows an inclusion of a gauge (i.e. BRST) invariant potential with a quartic renormalizable self-interaction term. Such kind of interaction term is forbidden for the fermionic Dirac fields in interaction with the (non-)Abelian gauge fields.

The contents of our present paper are organized as follows. In section 2, we recapitulate the key points of the nilpotent (anti-)BRST symmetry transformations for all the fields of an interacting $U(1)$ gauge theory in the Lagrangian formulation where there is an explicit coupling between the $U(1)$ gauge field and the complex scalar fields. Section 3 is devoted to the derivation of the on-shell nilpotent (anti-)BRST symmetry transformations for the appropriate fields of the above interacting gauge theory in the framework of the superfield formulation. In this derivation, the (anti-)chiral superfields are invoked for the gauge invariant restriction. The above specific superfields are defined on the $(4,1)$-dimensional (anti-)chiral super sub-manifold of the general $(4,2)$-dimensional supermanifold. The material of section 4 deals with the derivation of the off-shell nilpotent (anti-)BRST symmetry transformations for all the fields of the theory by exploiting a gauge invariant restriction on the general superfields of the $(4,2)$-dimensional supermanifold. An alternative version of this section is presented in the Appendix A. Finally, in section 5, we make some concluding remarks and point out a few future directions for further investigations.

\section{2 (Anti-)BRST symmetries: Lagrangian formalism}

To provide a brief synopsis of the salient features of the off-shell as well as on-shell nilpotent (anti-)BRST symmetries, we focus on the Lagrangian density of an interacting four $(3+1)$ dimensional $^{\dagger}(4 \mathrm{D}) U(1)$ gauge theory which describes a dynamically closed system of the charged complex scalar fields and U(1) gauge field. The (anti-)BRST invariant Lagrangian

\footnotetext{
${ }^{\dagger}$ We adopt here the conventions and notations such that the $4 \mathrm{D}$ flat Minkowski metric is: $\eta_{\mu \nu}=\operatorname{diag}$ $(+1,-1,-1,-1)$ and $\square=\eta^{\mu \nu} \partial_{\mu} \partial_{\nu}=\left(\partial_{0}\right)^{2}-\left(\partial_{i}\right)^{2}, F_{0 i}=\partial_{0} A_{i}-\partial_{i} A_{0}=E_{i} \equiv \mathbf{E}, F_{i j}=\epsilon_{i j k} B_{k}, B_{i} \equiv \mathbf{B}=$ $\frac{1}{2} \epsilon_{i j k} F_{j k},(\partial \cdot A)=\partial_{0} A_{0}-\partial_{i} A_{i}$ where $\mathbf{E}$ and $\mathbf{B}$ are the electric and magnetic fields, respectively and $\epsilon_{i j k}$ is the totally antisymmetric Levi-Civita tensor defined on the 3D (space) sub-manifold of the 4D spacetime manifold. Here the Greek indices: $\mu, \nu, \lambda \ldots=0,1,2,3$ correspond to the spacetime directions and Latin indices $i, j, k, \ldots=1,2,3$ stand only for the space directions on the Minkowski spacetime manifold.
} 
density of the above system, in the Feynman gauge, is $[7,20-22]$

$$
\begin{aligned}
\mathcal{L}_{b} & =-\frac{1}{4} F^{\mu \nu} F_{\mu \nu}+\bar{D}_{\mu} \phi^{*} D^{\mu} \phi-V\left(\phi^{*} \phi\right)+B(\partial \cdot A)+\frac{1}{2} B^{2}-i \partial_{\mu} \bar{C} \partial^{\mu} C \\
& \equiv \frac{1}{2}\left(\mathbf{E}^{2}-\mathbf{B}^{2}\right)+\bar{D}_{\mu} \phi^{*} D^{\mu} \phi-V\left(\phi^{*} \phi\right)+B(\partial \cdot A)+\frac{1}{2} B^{2}-i \partial_{\mu} \bar{C} \partial^{\mu} C
\end{aligned}
$$

where $V\left(\phi^{*} \phi\right)$ is the potential describing the quadratic and quartic interactions between the complex scalar fields $\phi$ and $\phi^{*} \ddagger$. The covariant derivatives on these fields, that are endowed with the electric charge $e$, are as given below

$$
D_{\mu} \phi=\partial_{\mu} \phi+i e A_{\mu} \phi \quad \bar{D}_{\mu} \phi^{*}=\partial_{\mu} \phi^{*}-i e A_{\mu} \phi^{*}
$$

In the Lagrangian density (2.1), the Nakanishi-Lautrup auxiliary field $B$ is required to linearize the gauge-fixing term $-\frac{1}{2}(\partial \cdot A)^{2}$. The Faddeev-Popov (anti-)ghost fields $(\bar{C}) C$ (with $C^{2}=\bar{C}^{2}=0, C \bar{C}+\bar{C} C=0$ ) are needed in the theory to maintain the "quantum" gauge (i.e. BRST) invariance and unitarity together at any arbitrary order of perturbative calculations (see, e.g. [24]). In the sense of the basic requirements of a canonical field theory, the Lagrangian density $\mathcal{L}_{b}$ describes a dynamically closed system of all the fields $\phi, \phi^{*}, C, \bar{C}$ and $A_{\mu}$ (see, e.g., [23]). It will be noted that the gauge field $A_{\mu}$ couples to the conserved matter current $J_{\mu}^{(c)} \sim\left(\phi^{*} D_{\mu} \phi-\phi \bar{D}_{\mu} \phi^{*}\right)$ to provide the interaction between $A_{\mu}$ and matter fields $\phi$ and $\phi^{*}$. This statement can be succinctly expressed as

$$
\begin{aligned}
\mathcal{L}_{b} & =-\frac{1}{4} F^{\mu \nu} F_{\mu \nu}+\partial_{\mu} \phi^{*} \partial^{\mu} \phi-i e A_{\mu}\left[\phi^{*} \partial_{\mu} \phi-\phi \partial_{\mu} \phi^{*}\right]+e^{2} A^{2} \phi^{*} \phi \\
& -V\left(\phi^{*} \phi\right)+B(\partial \cdot A)+\frac{1}{2} B^{2}-i \partial_{\mu} \bar{C} \partial^{\mu} C .
\end{aligned}
$$

The conservation of the matter current $J_{\mu}^{(c)}$ can be easily checked by exploiting the equations of motion $D_{\mu} D^{\mu} \phi=-\left(\partial V / \partial \phi^{*}\right), \bar{D}_{\mu} \bar{D}^{\mu} \phi^{*}=-(\partial V / \partial \phi)$ derived from the above Lagrangian densities. These Lagrangian densities respect the following off-shell nilpotent $\left(s_{(a) b}^{2}=0\right)$ and anticommuting $\left(s_{b} s_{a b}+s_{a b} s_{b}=0\right)\left(\right.$ anti-)BRST symmetry transformations $s_{(a) b} \S$ for the matter fields, gauge field and the (anti-)ghost fields, namely;

$$
\begin{array}{lcclcc}
s_{b} A_{\mu} & =\partial_{\mu} C & s_{b} C=0 & s_{b} \bar{C}=i B & s_{b} \phi=-i e C \phi \\
s_{b} \phi^{*}=+i e \phi^{*} C & s_{b} \mathbf{B}=0 & s_{b} B=0 & s_{b} \mathbf{E}=0 & s_{b}(\partial \cdot A)=\square C \\
s_{a b} A_{\mu}=\partial_{\mu} \bar{C} & s_{a b} \bar{C}=0 & s_{a b} C=-i B & s_{a b} \phi=-i e \bar{C} \phi \\
s_{a b} \phi^{*}=+i e \phi^{*} \bar{C} & s_{a b} \mathbf{B}=0 & s_{a b} B=0 & s_{a b} \mathbf{E}=0 & s_{a b}(\partial \cdot A)=\square \bar{C}
\end{array}
$$

The key points to be noted, at this stage, are (i) under the (anti-)BRST transformations, it is the kinetic energy term $\left(-\frac{1}{4} F^{\mu \nu} F_{\mu \nu}\right)$ of the gauge field $A_{\mu}$ which remains invariant.

\footnotetext{
${ }_{\ddagger}^{\ddagger}$ This potential can be chosen in the quartic polynomial form as: $V\left(\phi^{*} \phi\right)=\alpha^{2} \phi^{*} \phi+\beta\left(\phi^{*} \phi\right)^{2}$ for a renormalizable quantum field theory. Here $\alpha$ and $\beta$ are the parameters which could be chosen in different ways for different purposes (see, e.g. [23]). The key point to be noted is the fact that this potential remains invariant under the $U(1)$ gauge transformations as well as the (anti-)BRST symmetry transformations.

${ }^{\S}$ We follow here the notations and conventions adopted in $[21,22]$. In fact, the (anti-)BRST prescription is to replace the local gauge parameter by an anticommuting number $\eta$ and the (anti-)ghost fields $(\bar{C}) C$ which anticommute (i.e. $\eta C+C \eta=0, \eta \bar{C}+\bar{C} \eta=0$ ) and commute with all the fermionic (i.e.C $\bar{C}+\bar{C} C=$ $0, C^{2}=\bar{C}^{2}=0$, etc.) and bosonic fields, respectively. In its totality, the nilpotent $\left(\delta_{(A) B}^{2}=0\right)($ anti-) BRST transformations $\delta_{(A) B}$ are the product (i.e. $\left.\delta_{(A) B}=\eta s_{(a) b}\right)$ of $\eta$ and $s_{(a) b}$ where $s_{(a) b}^{2}=0$.
} 
This statement is true for 1-form (non-)Abelian gauge theories. For the above $U(1)$ gauge theory, as it turns out, it is the curvature term $F_{\mu \nu}$ itself that remains invariant under the (anti-)BRST transformations. (ii) In the mathematical language, the (anti-)BRST symmetry transformations owe their origin to the exterior derivative $d=d x^{\mu} \partial_{\mu}$ because the curvature term, owing its origin to the 2-form $F^{(2)}=d A^{(1)}$, is constructed from it and the 1 -form connection $A^{(1)}=d x^{\mu} A_{\mu}$. (iii) One can obtain the on-shell $(\square C=\square \bar{C}=0$ ) nilpotent $\left(\tilde{s}_{(a) b}^{2}=0\right)$ (anti-)BRST symmetry transformations $\tilde{s}_{(a) b}$ for the above theory from (2.4), by the substitution $B=-(\partial \cdot A)$, as given below

$$
\begin{array}{rlccc}
\tilde{s}_{b} A_{\mu} & =\partial_{\mu} C & \tilde{s}_{b} C=0 & \tilde{s}_{b} \bar{C}=-i(\partial \cdot A) & \tilde{s}_{b} \phi=-i e C \phi \\
\tilde{s}_{b} \phi^{*}=+i e \phi^{*} C & \tilde{s}_{b} \mathbf{E}=0 & \tilde{s}_{b} \mathbf{B}=0 & \tilde{s}_{b}(\partial \cdot A)=\square C \\
\tilde{s}_{a b} A_{\mu}=\partial_{\mu} \bar{C} & \tilde{s}_{a b} \bar{C}=0 & \tilde{s}_{a b} C=+i(\partial \cdot A) & \tilde{s}_{a b} \phi=-i e \bar{C} \phi \\
\tilde{s}_{a b} \phi^{*}=+i e \phi^{*} \bar{C} & \tilde{s}_{a b} \mathbf{B}=0 & \tilde{s}_{a b} \mathbf{E}=0 & \tilde{s}_{a b}(\partial \cdot A)=\square \bar{C} .
\end{array}
$$

The above local, infinitesimal, anticommuting and on-shell nilpotent transformations are the symmetry transformations for the following Lagrangian density

$$
\begin{aligned}
\tilde{\mathcal{L}}_{b} & =-\frac{1}{4} F^{\mu \nu} F_{\mu \nu}+\partial_{\mu} \phi^{*} \partial^{\mu} \phi-i e A_{\mu}\left[\phi^{*} \partial_{\mu} \phi-\phi \partial_{\mu} \phi^{*}\right]+e^{2} A^{2} \phi^{*} \phi \\
& -V\left(\phi^{*} \phi\right)-\frac{1}{2}(\partial \cdot A)^{2}-i \partial_{\mu} \bar{C} \partial^{\mu} C
\end{aligned}
$$

which is derived from (2.3) by the substitution $B=-(\partial \cdot A)$. (iv) In general, the above transformations can be concisely expressed in terms of the generic fields $\Omega(x), \tilde{\Omega}(x)$ and the conserved charges $Q_{(a) b}, \tilde{Q}_{(a) b}$, as

$$
s_{r} \Omega(x)=-i\left[\Omega(x), Q_{r}\right]_{ \pm} \quad \tilde{s}_{r} \tilde{\Omega}(x)=-i\left[\tilde{\Omega}(x), \tilde{Q}_{r}\right]_{ \pm} \quad r=b, a b
$$

where the local generic fields $\Omega=A_{\mu}, C, \bar{C}, B, \phi, \phi^{*}$ and $\tilde{\Omega}=A_{\mu}, C, \bar{C}, \phi, \phi^{*}$ are the fields of the Lagrangian densities (2.3) and (2.6). The $(+)-$ signs, as the subscripts on the square bracket $[,]_{ \pm}$, stand for the bracket to be an (anti)commutator for $\Omega, \tilde{\Omega}$ being (fermionic)bosonic in nature. The explicit forms of the conserved, anticommuting and nilpotent (anti-)BRST charges $Q_{r}, \tilde{Q}_{r},(r=b, a b)$ are not required for our present discussion but can be derived for the symmetry transformations (2.4) and (2.5)(Noether theorem).

\section{On-shell nilpotent symmetries: superfield formalism}

In this section, we first focus on the derivation of the on-shell nilpotent BRST symmetry transformations for all the fields and, later on, we derive the anti-BRST symmetry transformations for all the fields by invoking the potential and power of specific restrictions on the chiral and anti-chiral superfields (defined on the $(4,1)$-dimensional super sub-manifolds of the general $(4,2)$-dimensional supermanifold), respectively.

\subsection{On-shell nilpotent BRST symmetries: chiral superfields}


To obtain the on-shell nilpotent BRST symmetry $\tilde{s}_{b}$ transformations (2.5) for the basic fields of the Lagrangian density (2.6), first of all, we generalize the $4 \mathrm{D}$ basic fields $A_{\mu}, C, \bar{C}, \phi, \phi^{*}$ to the corresponding chiral $(\theta=0)$ superfields defined on the $(4,1)$-dimensional super sub-manifold of the general $(4,2)$-dimensional supermanifold. These chiral superfields can be expanded in terms of the basic fields and some secondary fields (e.g. $R_{\mu}, B_{1}, B_{2}, f_{1}, f_{2}^{*}$ ) as

$$
\begin{array}{ll}
\mathcal{B}_{\mu}^{(c)}(x, \bar{\theta})=A_{\mu}(x)+\bar{\theta} R_{\mu}(x) & \mathcal{F}^{(c)}(x, \bar{\theta})=C(x)+i \bar{\theta} B_{1}(x) \\
\mathcal{F}^{(c)}(x, \bar{\theta})=C(x)+i \bar{\theta} B_{2}(x) & \Phi_{(c)}(x, \bar{\theta})=\phi(x)+i \bar{\theta} f_{1}(x) \\
\Phi_{(c)}^{*}(x, \bar{\theta})=\phi^{*}(x)+i \bar{\theta} f_{2}^{*}(x) . &
\end{array}
$$

The noteworthy points, at this stage, are:

(i) The chiral superfields $\mathcal{B}_{\mu}, \Phi, \Phi^{*}$ are bosonic (i.e. $\left.\left(\mathcal{B}_{\mu}\right)^{2} \neq 0,(\Phi)^{2} \neq 0,\left(\Phi^{*}\right)^{2} \neq 0\right)$ in nature whereas the superfields $\mathcal{F}, \overline{\mathcal{F}}$ are fermionic [i.e. $\mathcal{F}^{2}=0,(\overline{\mathcal{F}})^{2}=0, \mathcal{F} \overline{\mathcal{F}}+\overline{\mathcal{F}} \mathcal{F}=0$ ].

(ii) In the limit $\bar{\theta} \rightarrow 0$, one retrieves the basic fields of the Lagrangian density (2.6).

(iii) The number of fermionic fields $C, \bar{C}, f_{1}, f_{2}^{*}, R_{\mu}$ do match with the number of bosonic fields $B_{1}, B_{2}, \phi, \phi^{*}, A_{\mu}$ on the right hand side of the above super expansions.

(iv) All the fields, on the r.h.s. of the above expansion, are function of the $4 \mathrm{D}$ coordinates $x^{\mu}$ only because they have been expanded along the $\bar{\theta}$-direction of the super sub-manifold.

The following gauge (i.e. BRST) invariant restriction ${ }^{\Uparrow}$ on the chiral superfields $\phi_{(c)}(x, \bar{\theta})$ and $\phi_{(c)}^{*}(x, \bar{\theta})$ (defined on the $(4,1)$-dimensional chiral super sub-manifold of the general $(4,2)$-dimensional supermanifold), namely;

$$
\Phi_{(c)}^{*}(x, \bar{\theta}) \mathcal{D}_{(c)} \mathcal{D}_{(c)} \Phi_{(c)}(x, \bar{\theta})=\phi^{*}(x) D D \phi(x)
$$

leads to the derivation of all the on-shell nilpotent BRST transformations $\tilde{s}_{b}$ quoted in (2.5). In the above, the covariant derivative $D \phi(x)=\left(d+i e A^{(1)}\right) \phi(x) \equiv d x^{\mu}\left(\partial_{\mu}+i e A_{\mu}\right) \phi(x)$ where the exterior derivative $d=d x^{\mu} \partial_{\mu}$ and 1-form connection $A^{(1)}=d x^{\mu} A_{\mu}$. These quantities, defined on the ordinary 4D spacetime manifold, are generalized to the $(4,1)$-dimensional chiral super sub-manifold of the general $(4,2)$-dimensional supermanifold, as

$$
\begin{aligned}
& \mathcal{D}_{(c)}=\left(\tilde{d}_{(c)}+i e \tilde{A}_{(c)}^{(1)}\right) \equiv d x^{\mu}\left(\partial_{\mu}+i e \mathcal{B}_{\mu}^{(c)}\right)+d \bar{\theta}\left(\partial_{\bar{\theta}}+i e \mathcal{F}^{(c)}\right) \\
& \tilde{d}_{(c)}=d x^{\mu} \partial_{\mu}+d \bar{\theta} \partial_{\bar{\theta}} \equiv d Z_{(c)}^{M} \partial_{M}^{(c)} \quad \tilde{A}_{(c)}^{(1)}=d Z_{(c)}^{M} A_{M}^{(c)} \equiv d x^{\mu} \mathcal{B}_{\mu}^{(c)}+d \bar{\theta} \mathcal{F}^{(c)}
\end{aligned}
$$

where $Z_{(c)}^{M}=\left(x^{\mu}, \bar{\theta}\right)$ is the chiral superspace variable, $\partial_{M}^{(c)}$ is the chiral partial derivative and $A_{M}^{(c)}=\left(\mathcal{B}_{\mu}^{(c)}, \mathcal{F}^{(c)}\right)$ is the chiral supermultiplet. The r.h.s. of (3.2) leads to the definition of

\footnotetext{
"It will be noted that there exists another gauge (i.e. the on-shell nilpotent BRST) invariant restriction on the chiral superfields defined on the $(4,1)$-dimensional chiral super sub-manifold (of the general $(4,2)$ dimensional supermanifold) that also leads to the derivation of the on-shell nilpotent BRST transformations $\tilde{s}_{b}$ of $(2.5)$. This restriction is: $\Phi_{c}(x, \bar{\theta}) \tilde{\overline{\mathcal{D}}}_{(c)} \tilde{\overline{\mathcal{D}}}_{(c)} \Phi_{(c)}^{*}(x, \bar{\theta})=\phi(x) \bar{D} \bar{D} \phi^{*}(x)$ where $\bar{D} \phi^{*}=d x^{\mu}\left(\partial_{\mu}-i e A_{\mu}\right) \phi^{*}$ and $\tilde{\overline{\mathcal{D}}}_{(c)}=d x^{\mu}\left(\partial_{\mu}-i e \mathcal{B}_{\mu}^{(c)}\right)+d \bar{\theta}\left(\partial_{\bar{\theta}}-i e \mathcal{F}^{(c)}\right)$. It is evident that the r.h.s. of this restriction is: $-i e \phi(x) F^{(2)} \phi^{*}(x)$. This is a gauge (i.e. BRST) invariant quantity because $\tilde{s}_{b}\left(\phi F_{\mu \nu} \phi^{*}\right)=0$ as can be seen by exploiting the on-shell nilpotent BRST symmetry transformations (2.5).
} 
the ordinary 2-form $F^{(2)}$ (as well as the field strength tensor $F_{\mu \nu}$ ) as:

$$
\phi^{*}(x) D D \phi(x)=i e \phi^{*}(x)\left(F^{(2)}\right) \phi(x) \equiv \frac{1}{2 !}\left(d x^{\mu} \wedge d x^{\nu}\right) \phi^{*}(x)\left(F_{\mu \nu}\right) \phi(x) .
$$

It is straightforward to check that the above quantity is a $U(1)$ gauge (i.e. BRST) invariant quantity because $\tilde{s}_{b} \phi=-i e C \phi, \tilde{s}_{b} F_{\mu \nu}=0, \tilde{s}_{b} \phi^{*}=+i e \phi^{*} C \Rightarrow \tilde{s}_{b}\left(\phi^{*} F_{\mu \nu} \phi\right)=0$.

The 1.h.s. of the gauge invariant restriction (3.2) would yield the coefficients of the 2-form differentials $\left(d x^{\mu} \wedge d x^{\nu}\right),\left(d x^{\mu} \wedge d \bar{\theta}\right),(d \bar{\theta} \wedge d \bar{\theta})$. It is evident, on the other hand, that the r.h.s. of the restriction yields only the coefficients of $\left(d x^{\mu} \wedge d x^{\nu}\right)$ (cf. (3.4)). The expanded version of the l.h.s. of the restriction in (3.2) is

$$
\begin{aligned}
& \left(d x^{\mu} \wedge d x^{\nu}\right) \Phi_{(c)}^{*}(x, \bar{\theta})\left[\left(\partial_{\mu}+i e \mathcal{B}_{\mu}^{(c)}\right)\left(\partial_{\nu}+i e \mathcal{B}_{\nu}^{(c)}\right)\right] \Phi_{(c)}(x, \bar{\theta})-(d \bar{\theta} \wedge d \bar{\theta}) \\
& \Phi_{(c)}^{*}(x, \bar{\theta})\left[\left(\partial_{\bar{\theta}}+i e \mathcal{F}^{(c)}\right)\left(\partial_{\bar{\theta}}+i e \mathcal{F}^{(c)}\right)\right] \Phi_{(c)}(x, \bar{\theta})+\left(d x^{\mu} \wedge d \bar{\theta}\right) \\
& \Phi_{(c)}^{*}(x, \bar{\theta})\left[\left(\partial_{\mu}+i e \mathcal{B}_{\mu}^{(c)}\right)\left(\partial_{\bar{\theta}}+i e \mathcal{F}^{(c)}\right)-\left(\partial_{\bar{\theta}}+i e \mathcal{F}^{(c)}\right)\left(\partial_{\mu}+i e \mathcal{B}_{\mu}^{(c)}\right)\right] \Phi_{(c)}(x, \bar{\theta})
\end{aligned}
$$

It is obvious that the coefficients of the 2-form differentials $(d \bar{\theta} \wedge d \bar{\theta}),\left(d x^{\mu} \wedge d \bar{\theta}\right)$ would be set equal to zero to maintain the sanctity of (3.2). Such an operation on the coefficient of the former, leads to

$$
-i \text { e } \Phi_{(c)}^{*}(x, \bar{\theta})\left(\partial_{\bar{\theta}} \mathcal{F}^{(c)}\right) \Phi_{(c)}(x, \bar{\theta})=0 .
$$

For $e \neq 0, \Phi_{(c)} \neq 0, \Phi_{(c)}^{*} \neq 0$, we obtain $\partial_{\bar{\theta}} \mathcal{F}^{(c)}=0$ which implies $B_{1}(x)=0$ in the expansion of $\mathcal{F}^{(c)}(x, \bar{\theta})$ in $(3.1)$. This shows that the reduced form (i.e. $\left.\mathcal{F}^{(c)}(x, \bar{\theta}) \rightarrow \mathcal{F}_{(r)}^{(c)}(x, \bar{\theta})\right)$ of the expansion for the fermionic chiral superfield $\mathcal{F}^{(c)}(x, \bar{\theta})$ is: $\mathcal{F}_{(r)}^{(c)}(x, \bar{\theta})=C(x)$. This leads to primarily a pair of consequences. First, to maintain the sanctity of the restriction (3.2), the chiral superfield $\mathcal{F}^{(c)}(x, \bar{\theta})$ becomes a local ordinary $4 \mathrm{D}$ field $C(x)$. Second, it implies that the on-shell nilpotent BRST transformations for the ghost field $C(x)$ is zero (i.e. $\left.\mathcal{F}_{(r)}^{(c)}(x, \bar{\theta})=C(x)+\bar{\theta}\left(\tilde{s}_{b} C(x)\right)\right)$. Setting the coefficient of $\left(d x^{\mu} \wedge d \bar{\theta}\right)$ equal to zero, ultimately, implies the following relationship between the chiral superfields:

$$
\partial_{\mu} \mathcal{F}_{(r)}^{(c)}=\partial_{\bar{\theta}} \mathcal{B}_{\mu}^{(c)} \Rightarrow R_{\mu}(x)=\partial_{\mu} C(x)
$$

when $e \neq 0, \Phi_{(c)} \neq 0, \Phi_{(c)}^{*} \neq 0$. Thus, the reduced form (i.e. $\left.\mathcal{B}_{\mu}^{(c)}(x, \bar{\theta}) \rightarrow \mathcal{B}_{\mu(r)}^{(c)}(x, \bar{\theta})\right)$ of the bosonic superfield, after the application of the restriction (3.2), becomes

$$
\mathcal{B}_{\mu(r)}^{(c)}(x, \bar{\theta})=A_{\mu}(x)+\bar{\theta} \partial_{\mu} C(x) \equiv A_{\mu}(x)+\bar{\theta}\left(\tilde{s}_{b} A_{\mu}(x)\right) .
$$

The above equation demonstrates the derivation of the on-shell nilpotent BRST symmetry transformation for the gauge field $A_{\mu}$ within the framework of superfield formalism.

Finally, we equate the coefficient of $\left(d x^{\mu} \wedge d x^{\nu}\right)$ from the 1.h.s. and r.h.s. of the gauge (i.e. BRST) invariant restriction (3.2). The precise form of this equality is

$$
\begin{aligned}
& \frac{1}{2} i e\left(d x^{\mu} \wedge d x^{\nu}\right) \Phi_{(c)}^{*}(x, \bar{\theta})\left(\partial_{\mu} \mathcal{B}_{\nu(r)}^{(c)}-\partial_{\nu} \mathcal{B}_{\mu(r)}^{(c)}\right) \Phi_{(c)}(x, \bar{\theta}) \\
& =\frac{1}{2} i e\left(d x^{\mu} \wedge d x^{\nu}\right) \phi^{*}(x)\left(\partial_{\mu} A_{\nu}-\partial_{\nu} A_{\mu}\right) \phi(x) .
\end{aligned}
$$


Using (3.8), it is straightforward to note that $\partial_{\mu} \mathcal{B}_{\nu(r)}^{(c)}-\partial_{\nu} \mathcal{B}_{\mu(r)}^{(c)}=\partial_{\mu} A_{\nu}-\partial_{\nu} A_{\mu}$. Ultimately, the above equality in (3.9) reduces to the following form ${ }^{\|}$

$$
\Phi_{(c)}^{*}(x, \bar{\theta}) \Phi_{(c)}(x, \bar{\theta})=\phi^{*}(x) \phi(x) .
$$

The above simplicity occurs because of the Abelian nature of the gauge theory under consideration. The same does not hold good for the non-Abelian interacting gauge theory where the gauge fields are group valued and, therefore, noncommutative in nature (see, e.g., [19] for details). The substitution of the expansions in (3.1) for the chiral superfields on the l.h.s. of (3.10) leads to the following condition

$$
\phi^{*}(x) f_{1}(x)+f_{2}^{*}(x) \phi(x)=0 .
$$

One of the simplest solutions to the above condition is the case where $f_{1}(x)$ is proportional to the basic field $\phi(x)$ and $f_{2}^{*}(x)$ is that of the $4 \mathrm{D}$ field $\phi^{*}(x)$. However, it should be noted that the secondary fields $f_{1}(x)$ and $f_{2}^{*}(x)$ are fermionic in nature whereas the complex scaler fields $\phi(x)$ and $\phi^{*}(x)$ are bosonic. For the precise value of the equality, one of the interesting choices (that makes sense) is:

$$
f_{1}(x)=-e C(x) \phi(x) \quad f_{2}^{*}(x)=+e \phi^{*}(x) C(x)
$$

where field $C(x)$ is the fermionic ghost field of the theory. This field has been brought in to make the above choice fermionic in nature for $f_{1}(x)$ and $f_{2}^{*}(x)$. The above choices, in some sense, are unique because the presence of the fermionic ghost field $C(x)$ is the only appropriate possibility in (3.12). The substitution of the above values into the super expansion (3.1) leads to the derivation of $\tilde{s}_{b}$ (cf. (2.5)) for the matter fields as given below

$$
\begin{aligned}
& \Phi_{(c)}^{(r)}(x, \bar{\theta})=\phi(x)+\bar{\theta}(-i e C(x) \phi(x)) \equiv \phi(x)+\bar{\theta}\left(\tilde{s}_{b} \phi(x)\right) \\
& \Phi_{(c)}^{*(r)}(x, \bar{\theta})=\phi^{*}(x)+\bar{\theta}\left(+i e \phi^{*}(x) C(x)\right) \equiv \phi^{*}(x)+\bar{\theta}\left(\tilde{s}_{b} \phi^{*}(x)\right) .
\end{aligned}
$$

It should be emphasized that, so far, we have not been able to determine the exact value of the secondary field $B_{2}(x)$ in terms of the basic fields of the theory by exploiting the gauge (i.e. BRST) invariant restriction (3.2). At this stage, the equation of motion $B=-(\partial \cdot A)$ derived from (2.1) (or (2.3)) comes to our rescue if we identify ${ }^{* *}$ the secondary field $B_{2}(x)$ with the Nakanishi-Lautrup auxiliary field $B(x)$. With this input, we obtain the on-shell nilpotent symmetry transformations for all basic fields of the theory as the expansion (3.1) can be re-expressed, in terms of $\tilde{s}_{b}$ (cf. (2.5)), as

$$
\begin{array}{rlr}
\mathcal{B}_{\mu(r)}^{(c)}(x, \bar{\theta}) & =A_{\mu}(x)+\bar{\theta}\left(\tilde{s}_{b} A_{\mu}(x)\right) & \mathcal{F}_{(r)}^{(c)}(x, \bar{\theta})=C(x)+\bar{\theta}\left(\tilde{s}_{b} C(x)\right) \\
\overline{\mathcal{F}}_{(r)}^{(c)}(x, \bar{\theta}) & =C(x)+\bar{\theta}\left(\tilde{s}_{b} \bar{C}(x)\right) & \Phi_{(c)}^{(r)}(x, \bar{\theta})=\phi(x)+\bar{\theta}\left(\tilde{s}_{b} \phi(x)\right) \\
\Phi_{(c)}^{*(r)}(x, \bar{\theta}) & =\phi^{*}(x)+\bar{\theta}\left(\tilde{s}_{b} \phi^{*}(x)\right) . &
\end{array}
$$

\footnotetext{
"It will be noted that the condition in (3.10) is a completely new relationship which can never originate from the horizontality condition alone. In fact, the horizontality condition, present in the usual superfield formalism [1-7], does not shed any light on the derivation of the (anti-)BRST symmetry transformations for the matter fields of a given interacting gauge theory, as pointed out earlier.

${ }^{* *}$ We lay stress, at this point of our argument, that we shall remain consistent with this identification [i.e. $B_{2}(x)=B(x) \equiv-(\partial \cdot A)$ ] throughout the body of our present text.
} 
The above equation provides the geometrical interpretation for the on-shell nilpotent BRST transformation $\tilde{s}_{b}$ (and the corresponding generator $\tilde{Q}_{b}$ ) as the translational generator along the Grassmannian direction $\bar{\theta}$ of the chiral super sub-manifold. In other words, the translation of the $(4,1)$-dimensional chiral superfields along the $\bar{\theta}$-direction of the chiral super sub-manifold results in the internal on-shell nilpotent BRST symmetry transformations $\tilde{s}_{b}$ for the corresponding basic 4D local fields of the Lagrangian density (2.6).

\subsection{On-shell nilpotent anti-BRST symmetries: anti-chiral superfields}

We invoke here the anti-chiral (i.e. $\bar{\theta}=0$ ) superfields $\mathcal{B}_{\mu}^{(a c)}, \mathcal{F}^{(a c)}, \overline{\mathcal{F}}^{(a c)}, \Phi_{(a c)}, \Phi_{(a c)}^{*}$, corresponding to the basic fields $A_{\mu}, C, \bar{C}, \phi, \phi^{*}$ of the $4 \mathrm{D}$ Lagrangian density (2.6), for the derivation of the anti-BRST symmetry transformations of (2.5). We expand the above superfields along the $\theta$-direction of the $(4,1)$-dimensional anti-chiral super sub-manifold. These expansions are

$$
\begin{array}{ll}
\mathcal{B}_{\mu}^{(a c)}(x, \theta)=A_{\mu}(x)+\theta \bar{R}_{\mu}(x) & \mathcal{F}^{(a c)}(x, \theta)=C(x)+i \theta \bar{B}_{1}(x) \\
\overline{\mathcal{F}}^{(a c)}(x, \theta)=C(x)+i \theta \bar{B}_{2}(x) & \Phi_{(a c)}(x, \theta)=\phi(x)+i \theta \bar{f}_{1}(x) \\
\Phi_{(a c)}^{*}(x, \theta)=\phi^{*}(x)+i \theta \bar{f}_{2}^{*}(x) &
\end{array}
$$

where the basic fields the Lagrangian density (2.6) are obtained in the limit $\theta \rightarrow 0$. In the above expansion, the fields $\bar{R}_{\mu}, \bar{B}_{1}, \bar{B}_{2}, \bar{f}_{1}, \bar{f}_{2}^{*}$ are the secondary fields which would be determined in terms of the basic fields of (2.6) by the imposition of the following gauge (i.e. (anti-)BRST) invariant restriction ${ }^{\dagger \dagger}$ on the anti-chiral superfields defined on the $(4$, 1)-dimensional super sub-manifold of the general (4,2)-dimensional supermanifold; namely;

$$
\Phi_{(a c)}^{*}(x, \theta) \tilde{\mathcal{D}}_{(a c)} \tilde{\mathcal{D}}_{(a c)} \Phi_{(a c)}(x, \theta)=\phi^{*}(x) D D \phi(x)
$$

where the anti-chiral covariant derivative $\tilde{\mathcal{D}}_{(a c)}$, on the $(4,1)$-dimensional anti-chiral super sub-manifold, is defined as

$$
\tilde{\mathcal{D}}_{(a c)}=\tilde{d}_{(a c)}+i e \tilde{A}_{(a c)}^{(1)} \equiv d x^{\mu}\left(\partial_{\mu}+i e \mathcal{B}_{\mu}^{(a c)}\right)+d \theta\left(\partial_{\theta}+i e \overline{\mathcal{F}}^{(a c)}\right) .
$$

Here $\tilde{d}^{(a c)}=d x^{\mu} \partial_{\mu}+d \theta \partial_{\theta}$ is the anti-chiral version of the super exterior derivative $\tilde{d}=$ $d x^{\mu} \partial_{\mu}+d \theta \partial_{\theta}+d \bar{\theta} \partial_{\bar{\theta}}$ and $\tilde{A}_{(a c)}^{(1)}=d x^{\mu} \mathcal{B}_{\mu}^{(a c)}+d \theta \overline{\mathcal{F}}^{(a c)}$ is the anti-chiral limit of the super 1-form connection $\tilde{A}^{(1)}=d x^{\mu} \mathcal{B}_{\mu}(x, \theta, \bar{\theta})+d \theta \overline{\mathcal{F}}(x, \theta, \bar{\theta})+d \bar{\theta} \mathcal{F}(x, \theta, \bar{\theta})$ that would be exploited in the next section (cf. Sec. 4) for the derivation of the off-shell nilpotent (anti-)BRST symmetry transformations for all the fields of the theory. It is straightforward to note that the r.h.s. of (3.16) defines the 2-form $F^{(2)}$ as: $i e \phi^{*}(x) F^{(2)} \phi(x) \equiv \frac{1}{2} i e\left(d x^{\mu} \wedge d x^{\nu}\right) \phi^{*}(x) F_{\mu \nu} \phi(x)$.

\footnotetext{
${ }^{\dagger \dagger}$ It is interesting to note that the combination $\Phi_{(a c)}(x, \theta) \tilde{\overline{\mathcal{D}}}_{(a c)} \tilde{\overline{\mathcal{D}}}_{(a c)} \Phi_{(a c)}^{*}(x, \theta)=\phi(x) \bar{D} \bar{D} \phi^{*}(x)$ is also a gauge (i.e. (anti-)BRST) invariant condition that could be imposed on the anti-chiral superfields of the $(4,1)$-dimensional super sub-manifold. This restriction also leads to the derivation of the on-shell nilpotent anti-BRST symmetry transformations for all the fields of the theory. The computational steps are similar to those connected with the present condition in (3.16).
} 
Let us focus on the explicit form of the 1.h.s. of (3.16). In terms of the 2-form differentials $\left(d x^{\mu} \wedge d x^{\nu}\right),\left(d x^{\mu} \wedge d \theta\right)$ and $(d \theta \wedge d \theta)$, the 1.h.s. can be written in its most lucid form as

$$
\begin{aligned}
& \left(d x^{\mu} \wedge d x^{\nu}\right) \Phi_{(a c)}^{*}(x, \theta)\left[\left(\partial_{\mu}+i e \mathcal{B}_{\mu}^{(a c)}\right)\left(\partial_{\nu}+i e \mathcal{B}_{\nu}^{(a c)}\right)\right] \Phi_{(a c)}(x, \theta)-(d \theta \wedge d \theta) \\
& \Phi_{(a c)}^{*}(x, \theta)\left[\left(\partial_{\theta}+i e \overline{\mathcal{F}}^{(a c)}\right)\left(\partial_{\theta}+i e \overline{\mathcal{F}}^{(a c)}\right)\right] \Phi_{(a c)}(x, \theta)+\left(d x^{\mu} \wedge d \theta\right) \Phi_{(a c)}^{*}(x, \theta) \\
& {\left[\left(\partial_{\mu}+i e \mathcal{B}_{\mu}^{(a c)}\right)\left(\partial_{\theta}+i e \overline{\mathcal{F}}^{(a c)}\right)-\left(\partial_{\theta}+i e \overline{\mathcal{F}}^{(a c)}\right)\left(\partial_{\mu}+i e \mathcal{B}_{\mu}^{(a c)}\right)\right] \Phi_{(a c)}(x, \theta) .}
\end{aligned}
$$

It is evident that the coefficient of $(d \theta \wedge d \theta)$ of the above equation would be set equal to zero because there is no such term on the r.h.s. of (3.16). The simplified version of the consequence of this statement can be expressed as

$$
-i e(d \theta \wedge d \theta) \Phi_{(a c)}^{*}(x, \theta)\left(\partial_{\theta} \overline{\mathcal{F}}^{(a c)}\right) \Phi_{(a c)}(x, \theta)=0
$$

For $e \neq 0, \Phi \neq 0, \Phi^{*} \neq 0$, we obtain the solution $\partial_{\theta} \overline{\mathcal{F}}^{(a c)}=0$. This leads to $\bar{B}_{2}=0$ in the expansion of $\overline{\mathcal{F}}^{(a c)}(x, \theta)$. Thus, the reduced form (i.e. $\overline{\mathcal{F}}^{(a c)} \rightarrow \overline{\mathcal{F}}_{(r)}^{(a c)}$ ) of this superfield can be re-expressed, in terms of the on-shell nilpotent operator $\tilde{s}_{a b}$, as

$$
\overline{\mathcal{F}}_{(r)}^{(a c)}(x, \theta)=\bar{C}(x)+0 \equiv \bar{C}(x)+\theta\left(\tilde{s}_{a b} \bar{C}(x)\right) .
$$

The above equation demonstrates the explicit derivation of the anti-BRST symmetry transformations for the anti-ghost field as $\tilde{s}_{a b} \bar{C}(x)=0$.

We now collect the coefficient of the 2-form differential $\left(d x^{\mu} \wedge d \theta\right)$ from the equation (3.18). In its simple form, it looks as follows

$$
i e\left(d x^{\mu} \wedge d \theta\right) \Phi_{(a c)}^{*}(x, \theta)\left(\partial_{\mu} \overline{\mathcal{F}}_{(r)}^{(a c)}-\partial_{\theta} \mathcal{B}_{\mu}^{(a c)}\right) \Phi_{(a c)}(x, \theta)=0
$$

It is obvious that for $e \neq 0, \Phi_{(a c)} \neq 0, \Phi_{(a c)}^{*} \neq 0$, we obtain the relationship $\partial_{\mu} \overline{\mathcal{F}}_{(r)}^{(a c)}=\partial_{\theta} \mathcal{B}_{\mu}^{(a c)}$ which implies that $\bar{R}_{\mu}(x)=\partial_{\mu} \bar{C}(x)$. Thus, the reduced form (i.e. $\left.\mathcal{B}_{\mu}^{(a c)}(x, \theta) \rightarrow \mathcal{B}_{\mu(r)}^{(a c)}(x, \theta)\right)$ of the bosonic superfield (corresponding to the gauge field $A_{\mu}$ ) is

$$
\mathcal{B}_{\mu(r)}^{(a c)}(x, \theta)=A_{\mu}(x)+\theta \partial_{\mu} \bar{C}(x) \equiv A_{\mu}(x)+\theta\left(\tilde{s}_{a b} A_{\mu}(x)\right) .
$$

The above equation establishes the exact derivation of the nilpotent anti-BRST symmetry transformation for the gauge field $A_{\mu}$ in the framework of the present superfield formalism. Collecting the coefficient of $\left(d x^{\mu} \wedge d x^{\nu}\right)$ from both the sides of the restriction (3.16), we obtain the following relationship

$$
\begin{aligned}
& \frac{1}{2} i e\left(d x^{\mu} \wedge d x^{\nu}\right) \Phi_{(a c)}^{*}(x, \theta)\left(\partial_{\mu} \mathcal{B}_{\nu(r)}^{(a c)}-\partial_{\nu} \mathcal{B}_{\mu(r)}^{(a c)}\right) \Phi_{(a c)}(x, \theta) \\
& =\frac{1}{2} i e\left(d x^{\mu} \wedge d x^{\nu}\right) \phi^{*}(x)\left(\partial_{\mu} A_{\nu}-\partial_{\nu} A_{\mu}\right) \phi(x) .
\end{aligned}
$$

Taking the help of (3.22), it is evident that $\partial_{\mu} \mathcal{B}_{\nu(r)}^{(a c)}-\partial_{\nu} \mathcal{B}_{\mu(r)}^{(a c)}=\partial_{\mu} A_{\nu}-\partial_{\nu} A_{\mu}$. Thus, the simplest form of the condition in (3.23), that emerges after a bit of simple algebra, is: $\Phi_{(a c)}^{*}(x, \theta) \Phi_{(a c)}(x, \theta)=\phi^{*}(x) \phi(x)$. It will be noted that this new relation is a gauge (i.e. $\mathrm{BRST}$ ) invariant relation and it cannot emerge from the usual horizontality condition. 
Inserting the expansion of (3.15) for the anti-chiral matter fields, we obtain the following relationship among the basic fields $\phi, \phi^{*}$ and the secondary fermionic fields $\bar{f}_{1}$ and $\bar{f}_{2}^{*}$, namely;

$$
\phi^{*}(x) \bar{f}_{1}(x)+\bar{f}_{2}^{*}(x) \phi(x)=0 .
$$

A close look at the above condition provides us the clue to choose the secondary fermionic fields $\bar{f}_{1}$ proportional to $\phi$ and $\bar{f}_{2}^{*}$ proportional to $\phi^{*}$. For the exact equality, we bring in the anti-ghost field $\bar{C}(x)$ of the theory which allows us to choose the following

$$
\bar{f}_{1}(x)=-e \bar{C}(x) \phi(x) \quad \bar{f}_{2}^{*}(x)=+e \phi^{*}(x) \bar{C}(x) .
$$

The insertion of the above values into the expansion (3.15) leads to the exact derivation of the anti-BRST symmetry transformations for the matter fields in the sense that the reduced form of the matter fields become: $\Phi_{(a c)}^{(r)}(x, \theta)=\phi(x)+\theta\left(\tilde{s}_{a b} \phi(x)\right), \Phi_{(a c)}^{*(r)}(x, \theta)=$ $\phi^{*}(x)+\theta\left(\tilde{s}_{a b} \phi^{*}(x)\right)$ in terms of the on-shell nilpotent anti-BRST symmetry transformations.

So far, we have not been able to determine the secondary field $\bar{B}_{1}(x)$ of the expansion of $\mathcal{F}^{(a c)}(x, \theta)$ (cf (3.15) in terms of the basic fields of the Lagrangian density (2.6). In fact, $\bar{B}_{1}(x)$ can be identified with the Nakanishi-Lautrup auxiliary field $B(x)$ with a minus sign (i.e. $\bar{B}_{1}(x)=-B(x)$ ). The reason behind this choice with a minus sign will become clear in the next section. We shall be consistent, however, with this specific choice throughout the body of our present text. At this stage, once again, the equation of motion $B(x)=$ $-(\partial \cdot A)(x)$ comes to our help. Thus, the exact expression for the secondary field $\bar{B}_{1}(x)$ becomes $\bar{B}_{1}(x)=+(\partial \cdot A)(x)$. Insertion of this value in the expansion of $\mathcal{F}^{(a c)}(x, \theta)$ (cf. $(3.15))$ reduces this superfield to the form $\mathcal{F}_{(r)}^{(a c)}(x, \theta)=C(x)+i \theta(\partial \cdot A) \equiv C(x)+\theta\left(\tilde{s}_{a b} C(x)\right)$. Ultimately, all the superfields in their reduced form (with the inputs from the on-shell nilpotent anti-BRST transformations (2.5)) can be re-expressed as

$$
\begin{array}{rlr}
\mathcal{B}_{\mu(r)}^{(a c)}(x, \theta) & =A_{\mu}(x)+\theta\left(\tilde{s}_{a b} A_{\mu}(x)\right) & \mathcal{F}_{(r)}^{(a c)}(x, \theta)=C(x)+\theta\left(\tilde{s}_{a b} C(x)\right) \\
\overline{\mathcal{F}}_{(a c)}^{(a c)}(x, \theta) & =C(x)+\theta\left(\tilde{s}_{a b} \bar{C}(x)\right) & \Phi_{(a c)}^{(r)}(x, \theta)=\phi(x)+\theta\left(\tilde{s}_{a b} \phi(x)\right) \\
\Phi_{(a c)}^{*(r)}(x, \theta) & =\phi^{*}(x)+\theta\left(\tilde{s}_{a b} \phi^{*}(x)\right) . &
\end{array}
$$

The above set of expansions provides the geometrical origin and interpretation for the onshell nilpotent anti-BRST symmetry transformation (and corresponding generator $\tilde{Q}_{a b}$ ) as the translational generator along $\theta$-direction of the anti-chiral super sub-manifold.

\section{Off-shell nilpotent (anti-)BRST symmetries: superfield formalism}

To obtain the off-shell nilpotent symmetry transformations (2.4) for all the fields of the theory in superfield formalism, we define the $4 \mathrm{D}$ ordinary interacting $U(1)$ gauge theory with complex scalar fields on a $(4,2)$-dimensional supermanifold parametrized by the general superspace coordinate $Z^{M}=\left(x^{\mu}, \theta, \bar{\theta}\right)$ where $x^{\mu}(\mu=0,1,2,3)$ are the four even spacetime coordinates and $\theta, \bar{\theta}$ are a pair of odd elements of a Grassmann algebra. On this 
supermanifold, one can define a set of superfields corresponding to the basic fields of the theory that are present in the Lagrangian density (2.6). The above superfields can be expanded in terms of these basic fields $A_{\mu}, C, \bar{C}, \phi, \phi^{*}$ and some secondary fields (along the Grassmannian directions of the (4, 2)-dimensional supermanifold) as [3,13,14]

$$
\begin{aligned}
\mathcal{B}_{\mu}(x, \theta, \bar{\theta}) & =A_{\mu}(x)+\theta \bar{R}_{\mu}(x)+\bar{\theta} R_{\mu}(x)+i \theta \bar{\theta} S_{\mu}(x) \\
\mathcal{F}(x, \theta, \bar{\theta}) & =C(x)+i \theta \bar{B}_{1}(x)+i \bar{\theta} B_{1}(x)+i \theta \bar{\theta} s(x) \\
\overline{\mathcal{F}}(x, \theta, \bar{\theta}) & =\bar{C}(x)+i \theta \bar{B}_{2}(x)+i \bar{\theta} B_{2}(x)+i \theta \bar{\theta} \bar{s}(x) \\
\Phi(x, \theta, \bar{\theta}) & =\phi(x)+i \theta \bar{f}_{1}(x)+i \bar{\theta} f_{1}(x)+i \theta \bar{\theta} b(x) \\
\Phi^{*}(x, \theta, \bar{\theta}) & =\phi^{*}(x)+i \theta \bar{f}_{2}^{*}(x)+i \bar{\theta} f_{2}^{*}(x)+i \theta \bar{\theta} b^{*}(x) .
\end{aligned}
$$

It is straightforward to note, in the above super expansion, that the local fields $R_{\mu}(x)$, $\bar{R}_{\mu}(x), C(x), \bar{C}(x), s(x), \bar{s}(x), f_{1}(x), \bar{f}_{1}(x), f_{2}^{*}(x), \bar{f}_{2}^{*}(x)$ are fermionic (anticommuting) and $A_{\mu}(x), S_{\mu}(x), B_{1}(x), \bar{B}_{1}(x), B_{2}(x), \bar{B}_{2}(x)$ are bosonic (commuting) in nature. In the above expansion, the bosonic- and fermionic degrees of freedom match and, in the limit: $\theta, \bar{\theta} \rightarrow 0$, we get back our basic fields $A_{\mu}, C, \bar{C}, \phi, \phi^{*}$ of the Lagrangian density (2.6)

To obtain the exact expressions for the secondary fields in terms of the basic fields (and auxiliary fields) of the theory, we invoke the following gauge (i.e. (anti-)BRST) invariant restriction on the suitable superfields of the general (4, 2)-dimensional supermanifold, namely;

$$
\Phi^{*}(x, \theta, \bar{\theta}) \tilde{\mathcal{D}} \tilde{\mathcal{D}} \Phi(x, \theta, \bar{\theta})=\phi^{*}(x) D D \phi(x)
$$

where the super covariant derivative $\tilde{\mathcal{D}}$ is defined, in terms of the super exterior derivative $\tilde{d}=d x^{\mu} \partial_{\mu}+d \theta \partial_{\theta}+d \bar{\theta} \partial_{\bar{\theta}}$ and the 1-form super connection $\tilde{A}^{(1)}=d x^{\mu} \mathcal{B}_{\mu}+d \theta \overline{\mathcal{F}}+d \bar{\theta} \mathcal{F}$, as

$$
\tilde{\mathcal{D}}=\tilde{d}+i e \tilde{A}^{(1)} \equiv d x^{\mu}\left(\partial_{\mu}+i e \mathcal{B}_{\mu}\right)+d \theta\left(\partial_{\theta}+i e \overline{\mathcal{F}}\right)+d \bar{\theta}\left(\partial_{\bar{\theta}}+i e \mathcal{F}\right) .
$$

It will be noted that, in the previous section, we have taken the limiting cases (i.e $\theta \rightarrow 0$ and $\bar{\theta} \rightarrow 0$ ) of the above definition for the chiral and anti-chiral super sub-manifolds. It is evident that the r.h.s. of (4.2) (i.e. $i$ e $\left.\phi^{*}(x) F^{(2)} \phi(x)\right)$ is a gauge (i.e. (anti-)BRST) invariant quantity on the supermanifold because $s_{(a) b}\left(\phi^{*} F^{(2)} \phi\right)=0$ (as is clear from the off-shell nilpotent (anti-)BRST transformations $s_{(a) b}$ quoted in equation (2.4)).

For our computations, it is important to express the l.h.s. of the gauge invariant restriction (4.2) in terms of the explicit 2-form differentials $\left(d x^{\mu} \wedge d x^{\nu}\right),(d \theta \wedge d \theta),(d \bar{\theta} \wedge d \bar{\theta}),(d \theta \wedge$ $d \bar{\theta}),\left(d x^{\mu} \wedge d \theta\right)$ and $\left(d x^{\mu} \wedge d \bar{\theta}\right)$. This is required so that we can compare the r.h.s. with the 1.h.s. of (4.2). All the terms, corresponding to these differentials together with their coefficients from the l.h.s. of the restriction (4.2), are explicitly written as follows

$$
\begin{aligned}
& \left(d x^{\mu} \wedge d x^{\nu}\right) \Phi^{*}\left(\partial_{\mu}+i e \mathcal{B}_{\mu}\right)\left(\partial_{\nu}+i e \mathcal{B}_{\nu}\right) \Phi \\
& -(d \theta \wedge d \theta) \Phi^{*}\left(\partial_{\theta}+i e \overline{\mathcal{F}}\right)\left(\partial_{\theta}+i e \overline{\mathcal{F}}\right) \Phi \\
& -(d \bar{\theta} \wedge d \bar{\theta}) \Phi^{*}\left(\partial_{\bar{\theta}}+i e \mathcal{F}\right)\left(\partial_{\bar{\theta}}+i e \overline{\mathcal{F}}\right) \Phi \\
& -(d \theta \wedge d \bar{\theta}) \Phi^{*}\left[\left(\partial_{\bar{\theta}}+i e \mathcal{F}\right)\left(\partial_{\theta}+i e \overline{\mathcal{F}}\right)+\left(\partial_{\theta}+i e \overline{\mathcal{F}}\right)\left(\partial_{\bar{\theta}}+i e \mathcal{F}\right)\right] \Phi \\
& +\left(d x^{\mu} \wedge d \theta\right) \Phi^{*}\left[\left(\partial_{\mu}+i e \mathcal{B}_{\mu}\right)\left(\partial_{\theta}+i e \overline{\mathcal{F}}\right)-\left(\partial_{\theta}+i e \overline{\mathcal{F}}\right)\left(\partial_{\mu}+i e \mathcal{B}_{\mu}\right)\right] \Phi \\
& +\left(d x^{\mu} \wedge d \bar{\theta}\right) \Phi^{*}\left[\left(\partial_{\mu}+i e \mathcal{B}_{\mu}\right)\left(\partial_{\bar{\theta}}+i e \mathcal{F}\right)-\left(\partial_{\bar{\theta}}+i e \mathcal{F}\right)\left(\partial_{\mu}+i e \mathcal{B}_{\mu}\right)\right] \Phi
\end{aligned}
$$


For algebraic convenience, first of all, it is useful to set equal to zero the coefficients of the differentials $(d \theta \wedge d \theta),(d \bar{\theta} \wedge d \bar{\theta})$ and $(d \theta \wedge d \bar{\theta})$ as these are not present on the r.h.s. of the restriction (4.2). The outcome of the above algebraic conditions are

$$
\begin{aligned}
& -i e(d \theta \wedge d \theta) \Phi^{*}\left(\partial_{\theta} \overline{\mathcal{F}}\right) \Phi=0 \quad-i e(d \bar{\theta} \wedge d \bar{\theta}) \Phi^{*}\left(\partial_{\bar{\theta}} \mathcal{F}\right) \Phi=0 \\
& -i e(d \theta \wedge d \bar{\theta}) \Phi^{*}\left(\partial_{\theta} \mathcal{F}+\partial_{\bar{\theta}} \overline{\mathcal{F}}\right) \Phi=0
\end{aligned}
$$

For $e \neq 0, \Phi \neq 0, \Phi^{*} \neq 0$, we obtain the following solutions

$$
\begin{aligned}
& \partial_{\theta} \overline{\mathcal{F}}=0 \Rightarrow \bar{B}_{2}(x)=0 \\
& \partial_{\bar{\theta}} \mathcal{F}=0 \Rightarrow B_{1}(x)=0 \quad s(x)=0 \\
& \partial_{\theta} \mathcal{F}+\partial_{\bar{\theta}} \overline{\mathcal{F}}=0 \Rightarrow B_{2}(x)+\bar{B}_{1}(x)=0 .
\end{aligned}
$$

The insertions of these values into the super expansion of $\mathcal{F}$ and $\overline{\mathcal{F}}$ (along with our earlier identifications: $B_{2}(x)=B(x), \bar{B}_{1}(x)=-B(x)$ ), imply the following reduced forms (i.e. $\left.\mathcal{F}(x, \theta, \bar{\theta}) \rightarrow \mathcal{F}_{(r)}(x, \theta), \overline{\mathcal{F}}(x, \theta, \bar{\theta}) \rightarrow \overline{\mathcal{F}}_{(r)}(x, \bar{\theta})\right)$ of the superfield expansions

$$
\begin{aligned}
& \mathcal{F}_{(r)}(x, \theta)=C(x)-i \theta B(x) \equiv C(x)+\theta\left(s_{a b} C(x)\right) \\
& \overline{\mathcal{F}}_{(r)}(x, \bar{\theta})=\bar{C}(x)+i \bar{\theta} B(x) \equiv \bar{C}(x)+\bar{\theta}\left(s_{b} \bar{C}(x)\right) .
\end{aligned}
$$

The above equation imply (i) the derivation of the off-shell nilpotent (anti-)BRST symmetry transformations for the ghost and anti-ghost fields of the theory under consideration, (ii) the characteristic features of the superfields $\overline{\mathcal{F}}$ and $\mathcal{F}$ as the chiral and anti-chiral in nature after the application of the restriction (4.2), and (iii) the choices made in the previous section are correct because it can be seen that if $B_{2}(x)=B(x)$, the relation $B_{2}(x)+\bar{B}_{1}(x)=0$ implies that $\bar{B}_{1}(x)=-B(x)$. These results, which have been discussed above, are exactly same as that derived due to the application of the horizontality condition of the usual superfield formalism on the (4, 2)-dimensional supermanifold (see, e.g, [3,8,9] for details).

We now collect the coefficients of the 2-form differentials $\left(d x^{\mu} \wedge d \theta\right)$ and $\left(d x^{\mu} \wedge d \bar{\theta}\right)$. These are, naturally, to be set equal to zero. The consequences are listed below

$$
\begin{aligned}
& +i e\left(d x^{\mu} \wedge d \theta\right) \Phi^{*}\left(\partial_{\mu} \overline{\mathcal{F}}_{(r)}-\partial_{\theta} \mathcal{B}_{\mu}\right) \Phi=0 \\
& +i e\left(d x^{\mu} \wedge d \bar{\theta}\right) \Phi^{*}\left(\partial_{\mu} \mathcal{F}_{(r)}-\partial_{\bar{\theta}} \mathcal{B}_{\mu}\right) \Phi=0
\end{aligned}
$$

It will be noted here that the reduced values $(4.7)$ of the superfields $\mathcal{F}$ and $\overline{\mathcal{F}}$ have been taken into account for the above computations. For $e \neq 0, \Phi \neq 0, \Phi^{*} \neq 0$, we obtain the following explicit and precise solutions to the above restrictions

$$
R_{\mu}(x)=\partial_{\mu} C(x) \quad \bar{R}_{\mu}(x)=\partial_{\mu} \bar{C}(x) \quad S_{\mu}(x)=\partial_{\mu} B(x) .
$$

The insertions of the above values into the expansion of $\mathcal{B}_{\mu}$ on the $(4,2)$-dimensional supermanifold, leads to the following reduced form of this superfield, namely;

$$
\mathcal{B}_{\mu(r)}(x, \theta, \bar{\theta})=A_{\mu}(x)+\theta\left(s_{a b} A_{\mu}(x)\right)+\bar{\theta}\left(s_{b} A_{\mu}(x)\right)+\theta \bar{\theta}\left(s_{b} s_{a b} A_{\mu}(x)\right)
$$


The above equation demonstrates the exact derivation of the off-shell nilpotent (anti-)BRST symmetry transformations $s_{(a) b}$ for the $U(1)$ gauge field $A_{\mu}(x)$. The above result is also same as the one derived due to the application of the horizontality condition alone.

We concentrate on the comparison of the coefficients of the 2-form differentials $\left(d x^{\mu} \wedge\right.$ $\left.d x^{\nu}\right)$, constructed with the help of spacetime variables alone, from the l.h.s. and r.h.s. of (4.2). It should be noted that we shall be taking into account the reduced form of the superfield $\mathcal{B}_{\mu}$ in our present computation. Ultimately, we obtain the following relationship

$$
\begin{aligned}
& \frac{1}{2} i e\left(d x^{\mu} \wedge d x^{\nu}\right) \Phi^{*}(x, \theta, \bar{\theta})\left(\partial_{\mu} \mathcal{B}_{\nu(r)}(x, \theta, \bar{\theta})-\partial_{\nu} \mathcal{B}_{\mu(r)}(x, \theta, \bar{\theta})\right) \Phi(x, \theta, \bar{\theta}) \\
& =\frac{1}{2} i e\left(d x^{\mu} \wedge d x^{\nu}\right) \phi^{*}(x)\left(\partial_{\mu} A_{\nu}(x)-\partial_{\nu} A_{\mu}(x)\right) \phi(x) .
\end{aligned}
$$

It can be checked that $\partial_{\mu} \mathcal{B}_{\nu(r)}-\partial_{\nu} \mathcal{B}_{\mu(r)}=\partial_{\mu} A_{\nu}-\partial_{\nu} A_{\mu}$. Furthermore, the Abelian nature of all the fields in the above equation allows us to cancel the gauge field part from the l.h.s. and r.h.s. of (4.11). This entails upon the above equation to reduce to

$$
\Phi^{*}(x, \theta, \bar{\theta}) \Phi(x, \theta, \bar{\theta})=\phi^{*}(x) \phi(x)
$$

where we have taken $e \neq 0, A_{\mu} \neq 0$ into consideration. We lay emphasis on the fact that the new relationship (4.12) is a gauge invariant condition which cannot be obtained from the application of the horizontality condition alone. The substitution of the expansions for the matter superfields (cf. (4.1)) leads to the following form for the l.h.s

$$
\begin{aligned}
& \phi^{*} \phi+i \theta\left(\phi^{*} \bar{f}_{1}+\bar{f}_{2}^{*} \phi\right)+i \bar{\theta}\left(\phi^{*} f_{1}+f_{2}^{*} \phi\right) \\
& +i \theta \bar{\theta}\left(\phi^{*} b+b^{*} \phi+i f_{2}^{*} \bar{f}_{1}-i \bar{f}_{2}^{*} f_{1}\right) .
\end{aligned}
$$

Equating the above expressions with the r.h.s. of (4.12) leads to the following conditions

$$
\begin{aligned}
& \phi^{*} \bar{f}_{1}+\bar{f}_{2}^{*} \phi=0 \quad \phi^{*} f_{1}+f_{2}^{*} \phi=0 \\
& \phi^{*} b+b^{*} \phi+i f_{2}^{*} \bar{f}_{1}-i \bar{f}_{2}^{*} f_{1}=0 .
\end{aligned}
$$

It will be noted that, in the above, the coefficients of the $\theta, \bar{\theta}$ and $\theta \bar{\theta}$-directions of the above expansions, have been set equal to zero separately and independently. At this juncture, our knowledge of the previous section comes to our help. The following interesting choices

$$
\begin{aligned}
& f_{1}=-e C \phi \quad \bar{f}_{1}=-e \bar{C} \phi \quad f_{2}^{*}=+e \phi^{*} C \quad \bar{f}_{2}^{*}=+e \phi^{*} \bar{C} \\
& b=-i e(B+e \bar{C} C) \phi \quad b^{*}=+i e \phi^{*}(B+e C \bar{C})
\end{aligned}
$$

satisfy all the above conditions quoted in (4.14). The logical arguments in deducing the above solutions are same as in the previous section. The insertions of these values into the super expansion (4.1) for the matter superfields leads to the following reduced form

$$
\begin{aligned}
& \Phi^{(r)}(x, \theta, \bar{\theta})=\phi(x)+\theta\left(s_{a b} \phi(x)\right)+\bar{\theta}\left(s_{b} \phi(x)\right)+\theta \bar{\theta}\left(s_{b} s_{a b} \phi(x)\right) \\
& \Phi^{*(r)}(x, \theta, \bar{\theta})=\phi^{*}(x)+\theta\left(s_{a b} \phi^{*}(x)\right)+\bar{\theta}\left(s_{b} \phi^{*}(x)\right)+\theta \bar{\theta}\left(s_{b} s_{a b} \phi^{*}(x)\right) .
\end{aligned}
$$

The above equation demonstrates explicitly the derivation of the off-shell nilpotent and anticommuting (anti-)BRST symmetry transformations $s_{(a) b}$ for the matter fields of the theory in the framework of the augmented superfield formulation. 
Finally, the reduced form of the expansions, quoted for all the superfields in (4.1), can be re-expressed in terms of the off-shell nilpotent (anti-)BRST symmetry transformations of (2.4) in the following uniform fashion for all the superfields of the theory:

$$
\begin{aligned}
\mathcal{B}_{\mu(r)}(x, \theta, \bar{\theta}) & =A_{\mu}(x)+\theta\left(s_{a b} A_{\mu}(x)\right)+\bar{\theta}\left(s_{b} A_{\mu}(x)\right)+\theta \bar{\theta}\left(s_{b} s_{a b} A_{\mu}(x)\right) \\
\mathcal{F}_{(r)}(x, \theta, \bar{\theta}) & =C(x)+\theta\left(s_{a b} C(x)\right)+\bar{\theta}\left(s_{b} C(x)\right)+\theta \bar{\theta}\left(s_{b} s_{a b} C(x)\right) \\
\overline{\mathcal{F}}_{(r)}(x, \theta, \bar{\theta}) & =\bar{C}(x)+\theta\left(s_{a b} \bar{C}(x)\right)+\bar{\theta}\left(s_{b} \bar{C}(x)\right)+\theta \bar{\theta}\left(s_{b} s_{a b} \bar{C}(x)\right) \\
\Phi^{(r)}(x, \theta, \bar{\theta}) & =\phi(x)+\theta\left(s_{a b} \phi(x)\right)+\bar{\theta}\left(s_{b} \phi(x)\right)+\theta \bar{\theta}\left(s_{b} s_{a b} \phi(x)\right) \\
\Phi^{*(r)}(x, \theta, \bar{\theta}) & =\phi^{*}(x)+\theta\left(s_{a b} \phi^{*}(x)\right)+\bar{\theta}\left(s_{b} \phi^{*}(x)\right)+\theta \bar{\theta}\left(s_{b} s_{a b} \phi^{*}(x)\right) .
\end{aligned}
$$

It will be noted that, in the above expansion, the trivial transformations $s_{b} C=0, s_{a b} \bar{C}=0$ have been taken into account. The above form of the uniform expansion for all the superfields leads to the geometrical as well as physical interpretation for (i) the (anti-)BRST charges $Q_{(a) b}$ (and the symmetry transformations $\left(s_{(a) b}\right)$ they generate) as the generators (cf. (2.7)) of translations (i.e. $\operatorname{Lim}_{\bar{\theta} \rightarrow 0}(\partial / \partial \theta), \operatorname{Lim}_{\theta \rightarrow 0}(\partial / \partial \bar{\theta})$ ) along the Grassmannian directions of the six $(4,2)$-dimensional supermanifold, (ii) the nilpotency property of the (anti-)BRST symmetry transformations (and corresponding generators) as a couple of successive translations (i.e. $\left.(\partial / \partial \theta)^{2}=0,(\partial / \partial \bar{\theta})^{2}=0\right)$ along any particular Grassmannian direction of the supermanifold, (iii) the anticommutativity property $s_{b} s_{a b}+s_{a b} s_{b}=0$ (and/or $\left.Q_{b} Q_{a b}+Q_{a b} Q_{b}=0\right)$ as a similar kind of relationship (i.e. $\left.(\partial / \partial \theta)(\partial / \partial \bar{\theta})+(\partial / \partial \bar{\theta})(\partial / \partial \theta)=0\right)$ existing between the translation generators along the $\theta$ and $\bar{\theta}$-directions of the supermanifold, and (iv) the internal (anti-)BRST symmetry transformations for the 4D ordinary local field of a Lagrangian density as the translation of the corresponding superfield along the Grassmannian direction(s) of the supermanifold.

\section{Conclusions}

In our present investigation, we have provided a generalization of the celebrated horizontality condition of the usual superfield approach to BRST formalism [1-7]. This has been done primarily for a couple of reasons. First, as is well-known, the horizontality condition on a specifically chosen supermanifold is not a gauge (i.e. BRST) invariant restriction. Rather, it is intrinsically a gauge covariant restriction ${ }^{\ddagger \ddagger}$ because the curvature tensor of a non-Abelian gauge theory transforms covariantly under the $\mathrm{SU}(\mathrm{N})$ gauge transformation (which is also reflected in the corresponding BRST transformation on it). A physical quantity, however, has to be a gauge (i.e. BRST) invariant quantity. This is why, in our present endeavour, we have chosen the gauge invariant restrictions (cf. (3.2), (3.16), (4.2), (A.1)) on the matter superfields of the appropriately chosen supermanifolds. Second, the horizontality condition does not shed any light on the (anti-)BRST symmetry transformations associated with the matter fields of a given interacting gauge theory where there is an ex-

\footnotetext{
¥†For an Abelian gauge theory, this restriction becomes a gauge invariant restriction. In general, this condition is a gauge covariant restriction on the gauge superfield (defined on a suitably chosen supermanifold) and, therefore, it is not a BRST invariant restriction on the above superfield.
} 
plicit coupling between the gauge field and matter fields. However, in our present attempt, we have chosen restrictions on the matter superfields of the suitably chosen supermanifolds in such a way that they enable us to determine the exact nilpotent (anti-)BRST symmetry transformations for the matter fields. Thus, our present and earlier attempts [18,19] do provide a theoretical basis for the generalization of the horizontality condition of the usual superfield approach to BRST formalism.

One of the key features of our gauge (i.e. BRST) invariant restrictions is the fact that they owe their origin to a pair of (super) covariant derivatives that operate on the matter (super) fields in unison. This specific unity of the two (super) covariant derivatives has intimate connection with the (super) curvature tensors of a given gauge theory. This is precisely the reason that the geometrical interpretations of the nilpotent (anti-)BRST symmetry transformations and their corresponding nilpotent generators, that emerge due to the application of the horizontality condition alone, remain intact under the gauge invariant restrictions of our present endeavour. Furthermore, it is very interesting to note that a single restriction on the matter superfields of the supermanifold allows us to obtain all the nilpotent symmetry transformations for all the fields of a given interacting gauge theory. Thus, our present generalization of the horizontality condition (owing its origin to the restriction on the super gauge fields alone) is very logical, economical and physically appealing. Our model being an interacting Abelian gauge theory, the (super) covariant derivatives are defined only on the matter (super) fields. The above (super) covariant derivatives do not exist for the Abelian (super) gauge as well as (super) (anti-)ghost fields.

In our present investigation, we have concentrated on the field theoretical model of the $4 \mathrm{D}$ interacting $U(1)$ gauge theory where there is an explicit coupling between the gauge field and complex scalar fields (and the gauge field itself). This model is interesting by itself because it allows the inclusion of a renormalizable quartic potential that is found to be gauge (i.e. BRST) invariant. This kind of potential cannot be included in an interacting 4D (non-)Abelian gauge theory with the fermionic Dirac fields. Furthermore, this field theoretic model allows discussions connected with the spontaneous symmetry breaking, Goldstone theorem, Higgs mechanism, etc., which are very useful in the context of the gauge theory of the standard model of electro-weak unification (see, e.g., [22-24]). Thus, to put our earlier ideas $[18,19]$ on a firmer footing, it is essential to check the validity of those propositions in the context of our present field theoretical model. It would be very interesting future endeavour to apply the ideas of our present work and that of $[18,19]$ to the case of gravitational theories which resemble very much with the non-Abelian gauge theories [22]. In fact, the idea of horizontality condition has already been applied to gravitational theories by Delbourgo, Jarvis and Thompson (see, e.g., [4]). This issue is presently under investigation under our augmented superfield formalism and our results would be reported in our future publications [25].

\section{Appendix A}


To clarify the claims made in the footnotes before the equations (3.2) and (3.16), we discuss here, in a concise fashion, the derivation of the off-shell nilpotent symmetry transformations from an alternate version of the restriction (4.2) imposed on the matter superfields of the $(4,2)$-dimensional supermanifold. This gauge (i.e. BRST) invariant restriction is

$$
\Phi(x, \theta, \bar{\theta}) \tilde{\overline{\mathcal{D}}} \tilde{\overline{\mathcal{D}}} \Phi^{*}(x, \theta, \bar{\theta})=\phi(x) \bar{D} \bar{D} \phi^{*}(x)
$$

where $\tilde{\overline{\mathcal{D}}}=d x^{\mu}\left(\partial_{\mu}-i e \mathcal{B}_{\mu}\right)+d \theta\left(\partial_{\theta}-i e \overline{\mathcal{F}}\right)+d \bar{\theta}\left(\partial_{\bar{\theta}}-i e \mathcal{F}\right)$. It is evident that the r.h.s. of the above gauge invariant condition is: $-\frac{1}{2} i e\left(d x^{\mu} \wedge d x^{\nu}\right) \phi(x)\left(\partial_{\mu} A_{\nu}-\partial_{\nu} A_{\mu}\right) \phi^{*}(x)$. To compare this with the 1.h.s, it is essential to expand the l.h.s. in explicit form as given below

$$
\begin{aligned}
& \left(d x^{\mu} \wedge d x^{\nu}\right) \Phi^{*}\left(\partial_{\mu}-i e \mathcal{B}_{\mu}\right)\left(\partial_{\nu}-i e \mathcal{B}_{\nu}\right) \Phi \\
& -(d \theta \wedge d \theta) \Phi^{*}\left(\partial_{\theta}-i e \overline{\mathcal{F}}\right)\left(\partial_{\theta}-i e \overline{\mathcal{F}}\right) \Phi \\
& -(d \bar{\theta} \wedge d \bar{\theta}) \Phi^{*}\left(\partial_{\bar{\theta}}-i e \mathcal{F}\right)\left(\partial_{\bar{\theta}}-i e \mathcal{F}\right) \Phi \\
& -(d \theta \wedge d \bar{\theta}) \Phi^{*}\left[\left(\partial_{\bar{\theta}}-i e \mathcal{F}\right)\left(\partial_{\theta}-i e \overline{\mathcal{F}}\right)+\left(\partial_{\theta}-i e \overline{\mathcal{F}}\right)\left(\partial_{\bar{\theta}}-i e \mathcal{F}\right)\right] \Phi \\
& +\left(d x^{\mu} \wedge d \theta\right) \Phi^{*}\left[\left(\partial_{\mu}-i e \mathcal{B}_{\mu}\right)\left(\partial_{\theta}-i e \overline{\mathcal{F}}\right)-\left(\partial_{\theta}-i e \overline{\mathcal{F}}\right)\left(\partial_{\mu}-i e \mathcal{B}_{\mu}\right)\right] \Phi \\
& +\left(d x^{\mu} \wedge d \bar{\theta}\right) \Phi^{*}\left[\left(\partial_{\mu}-i e \mathcal{B}_{\mu}\right)\left(\partial_{\bar{\theta}}-i e \mathcal{F}\right)-\left(\partial_{\bar{\theta}}-i e \mathcal{F}\right)\left(\partial_{\mu}-i e \mathcal{B}_{\mu}\right)\right] \Phi
\end{aligned}
$$

Setting, first of all, the coefficients of $d \theta \wedge d \theta),(d \bar{\theta} \wedge d \bar{\theta})$ and $(d \theta \wedge d \bar{\theta})$ equal to zero, we obtain the following conditions

$$
\begin{aligned}
& \partial_{\theta} \overline{\mathcal{F}}=0 \Rightarrow \bar{B}_{2}(x)=0 \\
& \partial_{\bar{\theta}} \mathcal{F}=0 \Rightarrow B_{1}(x)=0 \\
& \partial_{\theta} \mathcal{F}+\partial_{\bar{\theta}} \overline{\mathcal{F}}=0 \Rightarrow \bar{B}_{1}(x)+B_{2}(x)=0
\end{aligned}
$$

when $e \neq 0, \Phi \neq 0, \Phi^{*} \neq 0$. Thus, according to our earlier choice, if $B_{2}(x)=B(x)$ then $\bar{B}_{1}(x)=-B(x)$. Insertions of these values into the expansions in (4.1) reduces the fermionic superfields $\mathcal{F}$ and $\overline{\mathcal{F}}$ to $\mathcal{F}_{(r)}$ and $\overline{\mathcal{F}}_{(r)}$. Similarly, setting the coefficients of the 2-form differentials $\left(d x^{\mu} \wedge d \theta\right)$ and $\left(d x^{\mu} \wedge d \bar{\theta}\right)$ equal to zero leads to the following conditions for $e \neq 0, \Phi \neq 0, \Phi^{*} \neq 0$, namely;

$$
\partial_{\mu} \overline{\mathcal{F}}_{(r)}=\partial_{\theta} \mathcal{B}_{\mu} \quad \partial_{\mu} \mathcal{F}_{(r)}=\partial_{\bar{\theta}} \mathcal{B}_{\mu}
$$

In the above, we substitute the reduced forms of the fermionic superfields $\mathcal{F}$ and $\overline{\mathcal{F}}$ which are exactly same as the ones listed in (4.7). The resulting relations, that are found between the secondary fields of the expansion for $\mathcal{B}_{\mu}$ superfield and the basic fields (as well as the auxiliary field) are exactly same as the ones given in (4.9).

Finally, we compare the coefficients of the 2-form differentials $\left(d x^{\mu} \wedge d x^{\nu}\right)$ that emerge from the 1.h.s. and r.h.s. of the restriction (A.1). In its explicit form, this equality is

$$
\begin{aligned}
& -\frac{1}{2} i e\left(d x^{\mu} \wedge d x^{\nu}\right) \Phi^{*}(x, \theta, \bar{\theta})\left(\partial_{\mu} \mathcal{B}_{\nu(r)}(x, \theta, \bar{\theta})-\partial_{\nu} \mathcal{B}_{\mu(r)}(x, \theta, \bar{\theta})\right) \Phi(x, \theta, \bar{\theta}) \\
& =-\frac{1}{2} i e\left(d x^{\mu} \wedge d x^{\nu}\right) \phi^{*}(x)\left(\partial_{\mu} A_{\nu}(x)-\partial_{\nu} A_{\mu}(x)\right) \phi(x)
\end{aligned}
$$

where $\mathcal{B}_{\mu(r)}$ is the reduced form of the bosonic superfield $\mathcal{B}_{\mu}$ of the expansion (4.1) where $R_{\mu}=\partial_{\mu} C, \bar{R}_{\mu}=\partial_{\mu} \bar{C}$ and $S_{\mu}=\partial_{\mu} B$ have been substituted. One obtains, ultimately, the 
same relationship between the matter superfields and ordinary matter fields as given in (4.12). After this, all the steps of computation are same as the ones given in the equations from (4.13) till (4.17). This establishes the fact that the alternative gauge (i.e. BRST) invariant conditions, that are mentioned in the footnotes before equations (3.2) and (3.16), are equally useful in obtaining the nilpotent (anti-)BRST symmetry transformations for all the fields of the interacting $U(1)$ gauge theory where there is an explicit coupling between $A_{\mu}$ field and matter fields $\phi$ and $\phi^{*}$. In fact, we conclude, after some observations, that the algebraic computations of all the steps, for the alternative versions of the gauge (i.e. BRST) invariant restrictions, are exactly same as the ones given in the body of our present text except that one has to replace $e$ by $-e($ i.e. $e \rightarrow-e)$ in all the relevant equations.

\section{Acknowledgements}

Very useful suggestions and critical comments by our esteemed referees as well as the adjudicator are gratefully acknowledged.

\section{References}

[1] Thierry-Mieg J 1980 J. Math. Phys. 212834

Thierry-Mieg J 1980 Nuovo Cimento A 56396

[2] Quiros M, De Urries F J, Hoyos J, Mazon M L and Rodrigues E 1981 J. Math. Phys. 22767

[3] Bonora L and Tonin M 1981 Phys. Lett. B 9848 Bonora L, Pasti P and Tonin M 1981 Nuovo Cimento A 63353

[4] Delbourgo R and Jarvis P D 1981 J. Phys. A: Math. Gen. 15611 Delbourgo R, Jarvis P D and Thompson G 1982 Phys. Lett. B 10925

[5] Baulieu L and Thierry-Mieg J 1982 Nucl. Phys. B 197477 Baulieu L and Thierry-Mieg J 1982 Nucl. Phys. B 228259 Alvarez-Gaumé L and Baulieu L 1983 Nucl. Phys. B 212255

[6] Hwang D S and Lee C -Y 1997 J. Math. Phys. 3830

[7] Nakanishi N and Ojima I 1990 Covariant operator formalism of gauge theories and quantum gravity (World Scientific: Singapore)

[8] Malik R P 2004 Phys. Lett. B 584210 (Preprint hep-th/0311001)

[9] Malik R P 2004 Int. J. Geom. Methods Mod. Phys. 1467 (Preprint hep-th/0403230)

[10] Malik R P 2005 Int. J. Mod. Phys. A 204899 (Preprint hep-th/0402005) Malik R P 2005 Int. J. Mod. Phys. A 207285 (Erratum) (Preprint hep-th/0402005)

[11] Malik R P 2005 Mod. Phys. Lett. A 201767 (Preprint hep-th/0402123)

[12] Malik R P 2006 Eur. Phys. J. C 45513 (Preprint hep-th/0506109) 
[13] Malik R P 2004 J. Phys. A: Math. Gen. 375261 (Preprint hep-th/0311193)

[14] Malik R P 2006 Eur. Phys. J. C 48825 (Preprint hep-th/0510226)

[15] Malik R P 2006 Eur. Phys. J. C 47227 (Preprint hep-th/0507127)

[16] Malik R P and Mandal B P 2006 Eur. Phys. J. C 47219 (Preprint hep-th/0512334)

[17] Malik R P 2006 Superfield approach to exact and unique nilpotent symmetries, Appeared in the Proc. of the International Workshop on "Supersymmetries and Quantum Symmetries" (SQS:'05) (held at Bogoliubov Laboratory of Theoretical Physics, JINR, Dubna, Moscow Region, Russia, 27-31 July 2005) pp. 399-407, hep-th/0510029.

[18] Malik R P 2006 J. Phys. A: Math. Gen. 3910575 (Preprint hep-th/0510164)

[19] Malik R P 2006 An alternative to horizontality condition in superfield approach to BRST symmetries, hep-th/0603049 (To appear in Eur. Phys. J. C 49 (2007))

[20] Nishijima K 1986 Progress in quantum field theory Eds Ezawa H and Kamefuchi S (North Holand: Amsterdam)

For a review, see, e.g., Nishijima K 1996 Czech. J. Phys. 461

[21] Henneaux M and Teitelboim C 1992 Quantization of gauge systems (Princeton University Press: Princeton, NJ)

[22] Weinberg S 1996 The quantum theory of fields: Modern applications vol 2 (Cambridge University Press: Cambridge)

[23] Huang K 1982 Quarks, leptons and gauge fields (World Scientific: Singapore)

[24] Aitchison I J R and Hey A J G 1982 Gauge theories in particle physics: A practical introduction (Adam Hilger: Bristol)

[25] Malik R P, in preparation 